\title{
Effect of Mupirocin Treatment in Eradication of Staphylococcus aureus Nasal Carriage among Intensive Care Unit Personnel
}

Hami Kaboosi (PhD)

Department of Microbiology,

Ayatollah Amoli Branch, Islamic Azad

University, Amol, Iran

Abolfazl Khandan Del (MSc)

Department of Microbiology, 5th Azar Hospital, Golstan University of Medical Sciences, Gorgan, Iran

Ezzat allah Ghaemi (PhD)

Department of Microbiology, Faculty of Medicine, Golestan University of

Medical Sciences, Gorgan, Iran

Sepide Bakhshande Nosrat (MD)

Department of Obstetrics and

Gynecologic, Golestan University of

Medical Sciences, Gorgan, Iran

Ali Asghar Ayatollahi (MLD)

Department of Medical Laboratory

Science,School of Paramedical

Sciences, Golestan University of

Medical Sciences, Gorgan, Iran

Nastaran Golriz (BSc)

Department of Microbiology, 5th

AzarHospital, Golestan University of

Medical Sciences, Gorgan, Iran

Corresponding Author: Ezzat allah

Ghaemi

Email: eghaemi@yahoo.com

Tel: +989113711770

Adderess: Faculty of Medicine,

Golestan University of Medical

Sciences, Gorgan, Iran
ABSTRACT

Background and Objective: Staphylococcus aureus is the most common and important infectious agent. This bacterium can enter the bloodstream and cause some complications in the intensive care unit (ICU).This organism can frequently be found in the nose and transmitted by the carriers. This study aimed to determine the efficiency of eradicating S. aureus from the nose of ICU personnel in reducing the risk of $S$. aureus infections in the Panje Azar Hospital in Gorgan, Iran.

Methods: Sampling was done using sterile swabs collected from the anterior nasal passages.All samples containing Gram-positive cocci were sent to the laboratory of Faculty of Medicine for identification and evaluation of methicillin resistance. All $S$. aureus nasal carriers were treated with mupirocin ointment b.d for 5 days. The Samples were cultured again after five weeks to evaluate the eradication of $S$. auerus from the nose of subjects.

Results: 0verall, seven ICU personnel (11.7\%) were S. aureus carriers. Two isolates (3.3\%) were found as methicillin resistant using both methods of disc diffusion and PCR. The frequency distribution of positive cases indicated a significant difference in terms of work experience $(\mathrm{P}=0.012)$.

Conclusion: The findings show that treatment of carriers with $2 \%$ mupirocin topical ointment eradicates $S$. aureus from the nose. No $S$. aureus isolates was found in reculture of nasal samples. Treatment of healthy carriers can significantly reduce the risk of infections caused by the bacterium in the ICU.

Keywords: ICU, Mupirocin, Staphylococcus Aureus.

Received : 25 Nov 2014

Revised: 28 Feb 2015

Accepted: 01 Mar 2015

This paper should be cited as: Kaboosi H, Khandan Del A, Ghaemi EA, Bakhshande Nosrat S, Ayatollahi AA, Golriz N[Effect of Mupirocin Treatment in Eradication of Staphylococcus aureus Nasal Carriage among Intensive Care Unit Personnel ]. mljgoums. 2016; 10(2): 14-18 


\section{INTRODUCTION}

Staphylococcus is a genus of grampositive, catalase-positive, sporeless bacteria that often have no capsule and can grow in different environmental conditions. This organism is the second most common nosocomial pathogen. The bacterium has remained a public health concern and a challenge faced by physicians, due to its potential destructive power and increasing resistance against antibiotics. Staphylococcus aureus is able to cause a wide range of diseases: from very benign conditions such as folliculitis and furuncle to life-threatening disorders such as endocarditis, sepsis, staphylococcal toxic shock syndrome and staphylococcal scalded skin syndrome (1).

The infections in hospitalized patients are categorized into two groups of communityacquired infections and nosocomial infections. In recent years, methicillin-resistant $S$. aureus (MRSA) infections have been considered as one of the most important nosocomial infections (2).

There are two different scenarios in dealing with MRSA strains, multidrug-resistant species in hospitals that are usually accompanied by some risk factors or predisposing factors (such as catheters, surgery, hospitalization and living in elderly care centers).

The community-acquired MRSA with less resistance and more power to cause severe skin disease and progressive pneumonia. These species produce more invasive diseases. $S$. aureus is one of the most common causes of endemic and epidemic nosocomial infections that can lead to significant morbidity and moralities.

The number of nosocomial infections caused by Staphylococcus has significantly increased in recent years that may be due to using more invasive diagnostic and treatment procedures and lack of proper implementation of nosocomial infections control methods. The severity of complications of staphylococcal infections alone could explain the importance of investigating its control. The $S$. aureus is normally present in the anterior nares of the nose and approximately $25-30 \%$ of healthy individuals can be the carriers at any time. Some individuals such as physicians, nurses and hospital staff $(50 \%$, $70 \%$ and $90 \%$, respectively) are more likely to be the carriers (3). Usually, these people can also contaminate those around them, which is one of the main causes of infection transmission to patients. It also significantly increases the risk of nosocomial staphylococcal infections in these patients (4). Unfortunately, one of the most important problems is the risk of transmission of MSRA infections, which will increase the chance of infection with resistant objects. The methicillin-resistance gene is also genetically stable and the dissemination of the resistance gene is probably due to antibiotic selection pressure (5). Another important problem in hospitals is the constant risk of hospital microbs' resistance to new antibiotics. One of these problems is the possibility of presence of vancomycin-intermediate $S$. aureus strains and more importantly vancomycin-resistant $S$. aureus. Such infections can question the work of the best medical specialists, cause economic loss by long-term occupation of the hospital beds, and corrupt the main objective of macroeconomic policy of health systems in the world (providing access to public health facilities available in the country). About 2-7\% of hospitalized patients in the intensive care unit (ICU) become infected with nosocomial infections, leading to an average of 4-5 days of prolonged stay in hospital. The rate of these infections has increased by $36 \%$ over the past two decades (6). The incidence of nosocomial infections in Iran has been reported over 25\% (7). It is necessary to study antibiotic resistance in $S$. aureus due to the increasing emergence of resistant strains. Awareness toward the layout of antibiotic resistance can be a useful guide for treatment and control of nosocomial infections. It is necessary to conduct these types of studies periodically and separately by hospital infection-control groups at each center. This study aimed to determine the frequency of $S$. aureus nasal carriers among staff of ICU in panje Azar Hospital and then investigate the resistance pattern of $S$. aureus isolates, particularly MRSA. This study also investigated the effect of treating the carriers on the incidence of MRSA infections. The results of this study could pave the way for preventing the spread of MRSA infections in hospitals and can be extensible to the entire hospital if successful. 


\section{MATERIAL AND METHODS}

After explaining the objectives of the study and obtaining written consent, the anterior nasal swab samples were collected (two swabs from both nostrils) from ICU staff. The samples were immediately cultured on mannitol salt agar (MSA) to isolate $S$. aureus. The plates were transferred to microbiology laboratory of Golestan University of Medical Sciences in less than two hours, and then they incubated at $37{ }^{\circ} \mathrm{C}$ for $48-24$ hours. The colonies suspicious of $S$. aureus were identified by isolating the colonies grown on MSA with changed colors (from pink to yellow) due to mannitol fermentation.

After purification and Gram staining, catalase test, slide and tube coagulase tests and DNase test were performed for definitive diagnosis. Catalase, coagulase and DNase positives were identified as $S$.aureus (8). Resistance to methicillin was assessed using $30 \mu \mathrm{g}$ cefoxitin disks according to the CLSI 2013 guidelines (9).

For resistance against $30 \mu \mathrm{g}$ cefoxitin, inhibition zone of $\geq 23 \mathrm{~mm}$ and inhibition zone of $\leq 22 \mathrm{~mm}$ were considered as susceptible and resistant, respectively.

In addition, methicillin-resistance in all $S$. aureus isolates was evaluated by the PCR method. The following specific primers were used according to the PCR temperature program presented in the table below. mecA 1 (F) 5 -' AAAATCGATGGTAAAGGTTGGC- 3' mecA 2 (R)5' - AGTTCTGCAGTACCGGATTTGC - 3'
All personnel who were carrying S. aureus in their nose were treated with mupirocin ointment b.d for 5 days (10).

Mupirocin ointment (2\%) contains 2 grams of the antibiotic per 100 grams. The sampling was performed again after five weeks to evaluate the presence of $S$. aureus. The study was recorded in the Iranian registry of clinical trials (Code: IRCT 2014102519666N1). We analyzed the data by SPSS version 16 using $X^{2}$ test and considering P-value of less than 0.05.

\section{RESULTS}

Overall, 60 (45 females and 15 males with mean age of $30.9 \pm 5.9$ ) participated in the study from the total of 65 personnel and physicians working in the ICU during April and May 2014.

After completing demographic checklist, the sampling was carried out using sterile swabs from the anterior nasal passage. In total, seven (11.7\%) of them were the carriers of $S$. aureus and $88.3 \%$ were not. Frequency distribution of $\mathrm{S}$. aureus nasal carriers among the ICU staff based on gender male $(5 \%)$ in female $(6 / 7 \%)$, the difference was not statistically significant gender and Staphylococcus aureus carriers $(\mathrm{p}=0.2)$ (Table 2). Two isolates $(3.3 \%)$ were identified as MRSA using both methods of disc diffusion and PCR. There was no statistically significant difference between the number of $S$. aureus nasal carriers in terms of gender and education level.

Table 1-Thermocycler temperature protocol

\begin{tabular}{ccc}
\hline Duration & Temperature $\left({ }^{\circ} \mathrm{C}\right)$ & $\begin{array}{c}\text { Condition } \\
\text { Stage }\end{array}$ \\
\hline 30 seconds & & Denaturation \\
30 seconds & 55 & Annealing \\
60 seconds & 55 & Extension \\
5 minutes & 72 & Final extension \\
\hline
\end{tabular}

Table 2- Frequency distribution of $S$. aureus nasal carriers among the ICU staff of panje Azar Hospital based on gender and education level

\begin{tabular}{rlrc}
\hline - $_{\text {value }}$ & $\begin{array}{l}\text { Number of carriers in each } \\
\text { group (\%) }\end{array}$ & Number of subjects (\%) & $\begin{array}{l}\text { Number } \\
\text { Education level } \\
\text { and Gender }\end{array}$ \\
\hline 0.08 & $(\% 30) 3$ & $(\% 16 / 7) 10$ & Diploma or less \\
& $(\% 8) 4$ & $(\% 83 / 3) 50$ & Bachelor's degree \\
0.2 & $(\% 6 / 7) 4$ & 45 & Female \\
& $(\% 5) 3$ & 15 & Males \\
\hline
\end{tabular}


Figure 3- Frequency distribution of $S$. aureus nasal carriers among the ICU staff of panje Azar Hospital based on work experience

\begin{tabular}{rlrr}
\hline $\begin{array}{r}\text { P-value } \\
\text { each group (\%) }\end{array}$ & $\begin{array}{l}\text { Number of carriers in } \\
(\%)\end{array}$ & $\begin{array}{r}\text { Number } \\
\text { Work experience }\end{array}$ \\
\hline \multirow{2}{*}{0.012} & $(\% 3.7) 1$ & $(\% 45) 27$ & $<5$ \\
& $(\% 14.3)$ & $(\% 38) 23$ & $6-10$ \\
& $(\% 66.7) 4$ & $(\% 10) 6$ & $11-15$ \\
& $(\% 25) 1$ & $(\% 6.7) 4$ & $>15$ \\
\hline
\end{tabular}

This is due to the difference in the selective method of determining methicillin resistance. Thus, in the studies that they used disk diffusion, the frequency of MRSA was expected to be higher than the actual value (3). Hence, PCR or using 30-mcg cefoxitin is considered a more suitable method of determining exact cases of MRSA. In this study, resistance to methicillin was evaluated using 30 micrograms cefoxitin disks according to the 2013 CLSI guidelines, and PCR method was used to find the $m e c A$ gene.

The $S$. aureus carriers had $11.1 \pm 4.4$ years work experience, while those without $S$. aureus had $6.4 \pm 4.6$ years work experience. The highest frequency $(66.7 \%)$ was observed in the carriers with 11 to 15 years of work experience and the lowest frequency $(3.7 \%)$ in the ones with less than 5 years. The frequency distribution of positive cases indicates a significant difference in terms of work experience $(\mathrm{P}=0 / 012)$ (Table 2). Methicillinresistance evaluation of the seven $S$. aureus isolates using the disk diffusion test showed that two samples (MRSA) were resistant to cefoxitin (30 micrograms). Meanwhile, PCR results showed that both MRSA isolates found in the phenotypic method had the mecA gene. The results of re-cultivation of samples from $S$. aureus nasal carriers after 5 weeks showed that none of these individuals was positive and the use of Mupirocin helped them to eradicate the bacterium.

\section{DISCUSSION}

The disk diffusion test was used to investigate the frequency of MRSA isolates. Of the seven $S$. aureus isolates, two MRSA samples were resistant to cefoxitin (30 micrograms) and the frequency of MRSA in the study population was $3.3 \%$. The prevalence of MRSA in healthcare workers of health centers in the other parts of Iran has been reported differently ranging from 0 in Karmostaji study in 2005 to $30 \%$ in Ghasemian study in 2002 (2). The results showed that the use of mupirocin ointment and the emphasis on hand washing could significantly reduce the number of $S$. aureus. Sarmadian et al. study in 2004 at Valiasr Hospital showed that intranasal application of mupirocin for five days in $19 \mathrm{~S}$. aureus carriers could eradicate the bacterium in 17 $(89.5 \%)$ and no recurrence was observed in $87 \%$ of them after five weeks (4).

In the present study, mupirocin treatment was effective in all seven carriers, with no recurrence observed in the fifth week. Kalmeijer et al. study in 2002 showed the efficacy of $83.5 \%$ for treatment of $S$. aureus nasal carriers referred to the hospital for surgery. It was found that the risk of infection in deep tissues of the surgical site in Mupirocin-treated patients was five times less than the control group (11). In a large scale study, $91 \%$ successful eradication of $S$. aureus carriers with Mupirocin has been reported (12). Different results have been reported on the effectiveness of eradicating carriers in prevention of surgical, orthopedic and cardiac surgery wound infections, while Gernaat and Kluytmans studies clearly demonstrated that treatment with Mupirocin could significantly decrease postoperative infections $(13,14)$.

The results of the present study confirms the findings of the two mentioned studies, and well demonstrates the strong effect of treating carriers on preventing infections in the ICU. This indicates the importance of this drug combination in controlling $S$. aureus infections, especially MRSA infections. 


\section{CONCLUSION}

In this study, the low number of carriers in the ICU wards is considered a positive point that can have an important role in the control of nosocomial infections in Gorgan, since ICUs are one of the main sources of nosocomial infections dissemination.

\section{ACKNOWLEDGMENTS}

This paper was compiled on the basis of a thesis titled "Effect of identification and treatment of MRSA carriers on prevention of nosocomial infections in ICU of the panje Azar hospital in Gorgan", approved by the

\section{REFERENCES}

1.Rashidian M, TaherPour A, Goodarzi S. Nasal Carrier Rates and Antibiotic Resistance of Staphylococcus Aureus Isolates of Beasat Hospital Staff. Scientific Journalof KurdistanUniversityof Medical Sciences.2002;6(21):1-6.

2. Karmostaji A, Moradi N, Boushehri E, Jahed M, Dadsetan B, Sanginabadi F, et al. Nasal carrier rates and antibiogrm pattern of staphylococcus aureus strains isolated from hospital staff in teaching hospitals in Bandar Abbas. Hormozgan Medical Journal. 2008; 12(2):95-101.

3.Vaez H, Ghazi Saeed K, Moradi A, Tabaraei A,Khodabakhshi B, Bazouri M, et al. Antibiotic resistance pattern of methicillin resistant Staphylococcus aureus isolated from Health-educational centers of Gorgan, Iran, 2008-2009. Iran J Med Microbiol. 2010; 3(4): 31-36.

4. Sarmadian H, Didgar F, Abtahi, H. The comparison of topical nasal Mupirocin and single dose of oral Ciprofloxacin in treatment and reinfection of Staphylococcus Aureus carriers in personnel of Vali-easr hospital, Arak, 2004.Journal of ArakUniversityof Medical Sciences.2008;11(1): 40-44

5. Silva EC, Antas Md, Monteiro B Neto A, Rabelo MA, Melo FL, Maciel MA. Prevalence and Risk Factors for Staphylococcus aureus in Health-Care Workers at a University Hospital of Recife-PE.Braz J Infect Dis. 2008; 12(6): 504-8.

6. Steed CJ.Common infections acquired in the hospitals.Nurse Clin of North America. 1999; 34(2): 443-60.

7. Abdollahi AA, RahmaniH, KhodabakhshiB, BehnampourN. Assessment of level of knowledge, attitude and practice of employed nurses to nosocomial infection in teaching hospitals of Golestan University of Medical Sciences (2000). J GorganUni Med Sci. 2003; 5(1):80-86. [Persian]
Ethics Committee (509299302188).The authors would like to thank the Deputy of Research and Education of Golestan University of Medical Sciences for financial support. The help of all laboratory managers, staff of the ICU of the panje Azar Hospital and microbiology laboratory personnel of Faculty of Medicine at Golestan University of Medical Sciences is gratefully acknowledged.

\section{CONFLICT OF INTEREST}

All contributing authors declare no conflicts of interest.

8. Forbe BA,SahmDF, Weissfeld AS. Bailey \& Scotts Diagnostic Microbiology. $12^{\text {th }}$ ed. CV Mosby, New York. 2007; 172-213.

9. CLSI: Clinical and Laboratory Standards Institute. Performance standards for antimicrobial susceptibility testing; $23^{\text {th }}$ informational supplement. CLSI, Wayne, PA.M100-S21. 2013; 31(1): 161-165.

10.Bertman G, Katzung MD. Basic Pharmacology.NewYork: Mc Grow-Hill. 2011; 793-802.

11.Kalmeijer MD, Coertjens H, van Nieuwland-Bollen PM, Bogaers-Hofman D, de Baere GA, Stuurman A, et al. Surgical Site Infections in Orthopedic Surgery: The Effect of Mupirocin NasalOintment in a Double-Blind, Randomized, Placebo-Controlled Study. Clin Infect Dis. 2002;35(4):353-8.

12. Doebbeling BN, Breneman DL, Neu HC, Aly R, Yangco BG, Holley HP Jr, et al. Elimination of $S$. aureus nasal carriage in health care workers: analysis of six clinical trials with calcium mupirocin ointment The Mupirocin Collaborative Study Group. Clin Infect Dis. 1993; 17(3):466-74.

13. Kluytmans JA, Mouton JW, VandenBergh MF, Manders MJ, Maat AP, Wagenvoort JH, et al. Reduction of surgical site infections in cardiothoracic surgery by elimination of nasal carriage of S. aureus. Infect Control HospEpidemiol. 1996; 17(12):780-5.

14. Gernaat-van der Sluis AJ, Hoogenboom-Verdegaal AM, Edixhoven PJ, Spies-van Rooijen NH. Prophylactic mupirocin could reduce orthopedic wound infections. 1,044 patients treated with mupirocin compared with 1,260 historical controls. ActaOrthop Scand. 1998; 69(4): 412-4. 